Exercices Exercices de rhétorique

de rhétorique $\quad 4 \mid 2014$

Sur l'amplification

\title{
L'exagération de la faute : une technique propre à la lettre de remontrance
}

Cécile Lignereux

\section{(2) OpenEdition}

1 Journals

Édition électronique

URL : http://journals.openedition.org/rhetorique/349

DOI : 10.4000/rhetorique.349

ISSN : 2270-6909

Éditeur

UGA Éditions/Université Grenoble Alpes

Édition imprimée

ISBN : 978-2-84310-292-9

Référence électronique

Cécile Lignereux, "L'exagération de la faute : une technique propre à la lettre de remontrance »,

Exercices de rhétorique [En ligne], 4 | 2014, mis en ligne le 03 décembre 2014, consulté le 12 septembre 2020. URL : http://journals.openedition.org/rhetorique/349; DOI : https://doi.org/10.4000/rhetorique. 349

Ce document a été généré automatiquement le 12 septembre 2020.

\section{(c) () (ㅇ)}

Les contenus de la revue Exercices de rhétorique sont mis à disposition selon les termes de la Licence Creative Commons Attribution - Pas d'Utilisation Commerciale - Partage dans les Mêmes Conditions 4.0 International. 


\title{
L'exagération de la faute : une technique propre à la lettre de remontrance
}

\author{
Cécile Lignereux
}

1 Face aux difficultés financières auxquelles la comtesse de Grignan se voit confrontée, Mme de Sévigné accumule les conseils visant à orienter les décisions que sa fille est amenée à prendre, au moyen de séquences délibératives dont l'épistolière tente systématiquement d'adoucir le caractère intrusif grâce à toutes sortes de précautions oratoires ${ }^{1}$, conformément aux recommandations des traités de savoir-vivre en général et des manuels épistolaires en particulier ${ }^{2}$. Mais Mme de Sévigné va au-delà des conseils et du délibératif. Mettant un point d'honneur à exprimer sa désapprobation à l'égard des conduites qu'elle juge répréhensibles, Mme de Sévigné n'hésite pas à formuler des objurgations qui relèvent nettement de la logique judiciaire - autant $d^{\prime}$ '« ennuyeuses prôneries» (3 juillet $1675:$ I, 747) et de «tirades de réflexions » (6 avril 1672: I, 4713) qui n'ont pas peu contribué à l'image, idéologiquement marquée, d'une mère de famille raisonnable, aussi économe que prudente dans la gestion de ses affaires ${ }^{4}$. Qu'elles soient perçues comme de touchantes preuves de tendresse et de sollicitude ou comme d'insupportables intrusions motivées par un amour abusif et tyrannique, les réprimandes de Mme de Sévigné n'ont pas manqué de susciter de nombreux commentaires qui, s'ils ont le mérite d'attirer l'attention sur les enjeux relationnels des réprobations maternelles, présentent l'inconvénient d'occulter aussi bien les normes psycho-affectives que les préconstruits socio-discursifs qui président à leur mise en discours. Or l'épistolière ne morigénerait pas sa fille pour ses dépenses excessives si elle ne s'y sentait autorisée, en vertu de la convergence de plusieurs données constitutives du contexte anthropologique dans lequel s'inscrit la correspondance.

2 D'une part, si Mme de Sévigné prend régulièrement le risque de manifester « un excès de zèle" (9 mars 1672: I, 455) en blâmant un laxisme qui ne cesse d'aggraver la situation financière de la maison des Grignan, c'est qu'elle met à profit ses prérogatives maternelles - sa bienveillance attentionnée face à une enfant qui semble manquer de 
prudence apparaissant d'autant plus légitime qu'elle s'adosse à des pratiques familiales courantes. D'autre part, le refus de Mme de Sévigné de dissimuler sa désapprobation à l'égard d'une «dissipation " ( $1^{\text {er }}$ février 1690 : III, 824) qui ne fait qu'accélérer la ruine des Grignan, ne saurait être réduit à l'un des symptômes d'un amour maternel d'une intensité exceptionnelle, tant il s'appuie sur les présupposés axiologiques qui sont au fondement d'une conception exigeante de l'amitié - les ouvrages de morale et de savoir-vivre dénonçant à l'unanimité la « complaisance muette » qui consiste à « laisser faire cent sotises" à ses amis "sans les en avertir ${ }^{5}$ ", aussi bien que l'« espece de cruauté de les abandonner à leur mauvaise conduite ${ }^{6} »$. Ainsi Mme de Sévigné considère-t-elle comme une "trahison » le fait de " ne pas blâmer ce qui est blâmable " (24 mai 1690 : III, 883). Enfin, les remontrances que formule la marquise à l'égard des « dépenses extraordinaires » (6 novembre $1680:$ III, 53) auxquelles son gendre et sa fille refusent de renoncer tant ils sont désireux, en dépit de leur déroute financière, de maintenir le prestige de leur maison, relèvent sans doute autant d'habitudes sociodiscursives que de mécanismes psychologiques. À une époque où les sentiments ne sauraient être communiqués en dehors de comportements langagiers normalisés, les répréhensions maternelles apparaissent moins comme d'incontrôlables affects (en vertu desquels la manière d'aimer jalouse et autoritaire de Mme de Sévigné la conduirait à s'immiscer dans le quotidien de sa fille ${ }^{7}$ ) que comme les composantes d'une pratique discursive codifiée - étant admis que dans le cadre d'une correspondance familière, c'est une faute que de ne pas faire bénéficier de son avis (défini par Furetière comme "avertissement, instruction qu'on donne à quelqu'un de quelque chose qu'il ignore, ou à quoy il ne prend pas garde $\left.{ }^{8} »\right)$ celui qui en a besoin ${ }^{9}$. De fait, la lettre de remonstrance prend place parmi les rituels effectifs de la civilité épistolaire, comme l'atteste le Secrétaire à la mode de Puget de la Serre - un manuel assurément moins prescriptif que descriptif, qui, renonçant aux typologies fondées sur les catégories rhétoriques pour mieux prendre acte des besoins et des usages épistolaires contemporains, se contente de répartir empiriquement les différentes sortes de lettres en deux catégories: les lettres d'affaires, qui «sont celles qui traitent de choses qui importent » et les lettres de compliment, qui « servent à contracter amitié avec quelcun, ou à l'entretenir ${ }^{10} »$. C'est parmi les lettres d'affaires que Puget de la Serre classe les lettres de remonstrance, dont il distingue deux sortes : celles qui peuvent procéder « tout ouvertement» en raison du rapport hiérarchique des correspondants, et celles qui « requierent plus d'artifice», caractérisées par l'abondance de stratagèmes oratoires destinés à ne pas froisser le destinataire ${ }^{11}$.

3 Ainsi donc, replacées à la fois dans le contexte des pratiques familiales présidant à la répartition des rôles discursifs entre mère et fille, dans le système de valeurs dans lequel s'enracinent les droits et les devoirs de l'amitié et dans le cadre des usages épistolaires, les réprimandes maternelles doivent être appréhendées moins comme la traduction d'affects symptomatiques d'un état passionnel que comme un comportement discursif mobilisant une dynamique psycho-affective et des rouages socio-linguistiques codifiés. Dans ces conditions, restituer aux réprimandes de Mme de Sévigné leur pleine portée au sein de l'interaction épistolaire implique de confronter les modalités de leur mise en discours aux normes d'expression de la lettre de remontrance - du moins dans sa version la moins formelle, ainsi présentée par Puget de la Serre ${ }^{12}$ :

On escrit des lettres de remonstrance à celui qui a commis quelque faute, afin de la lui faire recognoistre, ou l'induire à la reparer. 
Elles n'ont point besoin de tant d'invention, quand on a quelque autorité sur la personne qu'on reprend, ou qu'on ne se soucie point en quelle part il recevra la remonstrance qu'on lui fait. Car alors on peut proceder tout ouvertement, \& apres avoir descrit \& exaggeré sa faute, lui representer combien griefvement il a offensé Dieu, \& quel tort il a fait à sa reputation; puis l'exhorter à se retirer de ce vice \& changer de vie, ou à réparer le tort qu'il a fait à son prochain, \& choses semblables.

S'il paraît de bonne méthode d'inventorier les paramètres rhétoriques présentés comme constitutifs de ce sous-genre épistolaire et de s'en servir de grille de lecture pour analyser, au plus près des formes langagières, le fonctionnement des séquences de remontrance dans les lettres à Mme de Grignan, c'est que le va-et-vient entre la théorie et la pratique présente l'avantage d'attirer le regard autant sur les procédés que l'épistolière choisit de privilégier que sur ceux qu'elle refuse d'actualiser. Dans le contexte spécifique de la relation d'intimité affective qui l'unit à sa fille, il n'est guère étonnant que Mme de Sévigné se sente libre de ne pas recourir à la totalité des ressorts présentés comme constitutifs de la remontrance. C'est ainsi que les séquences de remontrance qui figurent au sein des lettres familières écrites deux à trois fois par semaine à Mme de Grignan n'adoptent pas la disposition rigide dont Puget de la Serre énumère les étapes - de l'établissement des faits ("apres avoir descrit...») à l'exhortation finale («puis l'exhorter à...») en passant par la phase proprement argumentative (« lui representer combien griefvement... »). Consciente que « si voulant retirer d'un faux pas vôtre ami qui s'égare, vous le faites avec aigreur, vos remontrances l'irriteront, \& ne le corrigeront pas ", et que " pour [le] ramener plus sûrement à son devoir, il ne faut pas que les réprimandes soient assaisonnées de reproches trop aigres, car elles font toûjours un mauvais effet ", Mme de Sévigné ne saurait formuler ses réprimandes sans une relative discrétion - étant admis que «la discretion doit assaisonner les reprimandes qu'un Père fait a ses enfans, s'il veut qu'elles leur soient utiles ${ }^{13}$ ». C'est pourquoi, à l'opposé aussi bien de la reproduction conformiste de protocoles cérémonieux que d'une impensable improvisation langagière, Mme de Sévigné n'exploite, pour formuler ses réprimandes, que les procédures congruentes à la relation de familiarité qui l'unit à sa correspondante. Ainsi les séquences de remontrance des lettres à Mme de Grignan, dont il est possible de mettre au jour cinq caractéristiques rhétoriques saillantes, constituent-elles une bonne illustration de la manière dont les épistoliers du Grand Siècle, tout en se pliant aux usages de la civilité, ont l'habitude non seulement de ne retenir des patrons discursifs en vigueur dans la société mondaine que les composantes qui leur paraissent adaptées à la situation d'énonciation, mais encore d'assouplir au gré de leurs besoins pragmatiques le caractère conventionnel des procédés habituellement préconisés.

\section{Une situation pragmatique}

Dans la mesure où, selon Puget de la Serre, « on escrit des lettres de remonstrance à celui qui a commis quelque faute ${ }^{14} »$, ce sous-genre épistolaire relève bien de la logique judiciaire. Que l'on préfère relier les réprimandes de Mme de Sévigné à une scandaleuse volonté d'emprise motivée par un amour étouffant ou à la sollicitude d'une mère soucieuse d'empêcher sa fille d'accélérer le désastre financier qui la guette, elles ont pour objet les agissements de Mme de Grignan que sa mère considère comme des erreurs de gestion. Certes, la comtesse est loin d'être la seule responsable de la banqueroute de la famille des Grignan, grevée de dettes depuis de longues années et incapable de réduire un train de vie indispensable à son prestige. Néanmoins, en 
refusant de faire des économies sur les dépenses somptuaires, le comte et la comtesse se privent effectivement du seul moyen de tenter de remédier à la faillite de leur maison. Il n'est donc pas étonnant que Mme de Sévigné pratique souvent l'accusation, tant s'accumulent ce que la marquise juge être des prodigalités aussi inutiles que dangereuses - qu'il s'agisse des sommes perdues au jeu ${ }^{15}$, des frais engendrés par l'entretien des équipages et des domestiques appartenant aux invités ${ }^{16}$, des achats (à crédit) destinés à assouvir les goûts de luxe du comte en matière d'ameublement ${ }^{17}$, de la dilapidation dont se rendent coupables les beaux-frères de la comtesse en entreprenant à Grignan d'ambitieux travaux qu'ils n'ont pas les moyens de financer ${ }^{18}$ ou encore des "dépenses inutiles » liées à de mauvaises habitudes de gestion ${ }^{19}$. Si les lettres à Mme de Grignan sont émaillées de répréhensions ${ }^{20}$, c'est bien parce que tous les paramètres définissant la situation de discours propre à la correspondance entre Mme de Sévigné et sa fille autorisent la marquise à pratiquer l'acte de langage propre au genre judiciaire, l'accusation - aussi bien la relation entre les interlocutrices (leur proximité affective) que le contexte (le désastre financiers des Grignan), le genre pratiqué (une correspondance familière) et les représentations collectives relatives à la position institutionnelle de l'épistolière (l'image stéréotypée du parent dont la sagesse et la bienveillance légitiment la franchise).

\section{Un but persuasif}

5 En assignant à la lettre de remontrance une double finalité («On escrit des lettres de remonstrance à celui qui a commis quelque faute, afin de la lui faire recognoistre, ou l'induire à la reparer $\left.{ }^{21} »\right)$, Puget de la Serre insiste sur ce qui constitue toute la complexité du dessein persuasif propre à la remontrance. De fait, le but des réprimandes est moins d'accuser que d'amender, moins de critiquer que de mettre en garde en ce qui concerne les agissements futurs - ce qui est le propre de la correction, qui consiste en une évocation du passé (les erreurs commises) tournée vers l'avenir (la conduite blâmée étant appelée à être rectifiée). Le dictionnaire de Furetière indique ainsi que la remontrance "est aussi une légere \& honneste correction ou avertissement qu'on fait en general, ou en particulier, pour advertir ou corriger de quelques defauts ${ }^{22}$ " (nous soulignons). Que l'objectif des remontrances soit moins de dénoncer une conduite répréhensible que de la réformer, ou plutôt de la dénoncer en vue de la réformer, les rapproche considérablement de la logique propre à ce que $\mathrm{P}$. Jacob analyse comme la lettre d'avertissement - qu'il classe dans le genre judiciaire : "C'est une espece de correction, qui approche de l'invective : mais qui est plus moderée. Les advertissemens [...] tendent plus au profit de celuy à qui l'on escrit qu'à son blâme ${ }^{23}$ ». C'est bien dans l'intrication entre le délibératif et le judiciaire que réside la spécificité de l'avertissement (comme le confirment les fluctuations taxinomiques des traités d'épistolographie ${ }^{24}$ ), qui consiste à la fois à accuser d'une faute (ce qui relève du judiciaire) et à orienter les décisions à venir (ce qui est le propre du délibératif). Dans la mesure où les réprimandes de Mme de Sévigné visent à faire prendre conscience de ses erreurs à Mme de Grignan dans le but de lui faire adopter une ligne de conduite moins nuisible à ses affaires, leur efficacité est largement déterminée par un éthos (compris comme la présentation de soi dans le discours) bienveillant ${ }^{25}$ - les réprobations maternelles, aussi désagréables qu'elles puissent paraître, ayant pour objectif de faire 
bénéficier Mme de Grignan d'une sagesse dont elle semble dépourvue, c'est-à-dire de l'aider et de lui être utile ${ }^{26}$.

\section{Une image de soi}

6 Pour pouvoir faire des remontrances qui soient légitimes, encore faut-il détenir, comme le précise Puget de la Serre, « quelque autorité sur la personne qu'on reprend ${ }^{27}$ ». L'autorité que manifeste Mme de Sévigné dans ses mises en garde s'enracine à la fois dans son statut maternel et dans son expérience. Telles sont en effet les deux données constitutives de l'« image préalable » dont bénéficie l'épistolière aux yeux de sa fille, et que ses remontrances ne manquent pas de solliciter, voire de consolider - étant entendu que «la représentation de la personne du locuteur antérieure à sa prise de parole, parfois dite éthos préalable ou prédiscursif, est souvent au fondement de l'image qu'il construit dans son discours ${ }^{28} »$. D'une part, si les séquences de remontrances des lettres à Mme de Grignan sont acceptables, c'est d'abord parce que Mme de Sévigné, en tant que mère de la comtesse, occupe une position de supériorité hiérarchique. Le droit de faire des remontrances est en effet l'apanage des personnes qui jouissent d'une supériorité sur leur interlocuteur ${ }^{29}$ - supériorité dont l'ascendant parental constitue justement le prototype, comme l'attestent, dans le sillage de la tradition rhétorique ${ }^{30}$, les définitions et les exemples des dictionnaires contemporains. C'est ainsi que le dictionnaire de Furetière, avant d'indiquer que remonstrer «se dit aussi d'un superieur à un inferieur, quand il l'advertit doucement de son devoir » (s. v. remonstrer, fin), donne comme exemple (s. v. remonstrance, fin) : "Une mere fait des remonstrances à sa fille, quand elle n'a pas gardé la bienseance, la modestie ». Quant au dictionnaire de l'Académie ${ }^{31}$, pour qui remontrance «se dit aussi Des avertissemens qu'un superieur donne à son inférieur, pour l'obliger à se corriger », il assortit sa définition de l'exemple « Remontrance paternelle ». D'autre part, si Mme de Sévigné se sent habilitée à faire des remontrances à Mme de Grignan, c'est aussi parce qu'elle fait preuve d'authentiques compétences en matière d'économie domestique - la marquise, épaulée par son oncle l'abbé de Coulanges ${ }^{32}$, ayant non seulement réussi à rétablir l'état de ses finances, sérieusement compromises par les excès de son époux, mais parvenant encore à gérer avec sagesse et prudence une fortune régulièrement menacée ${ }^{33}$. De fait, les réprimandes à Mme de Grignan sont d'autant plus acceptables qu'elles ne manquent pas de réactiver l'image de gestionnaire scrupuleuse dont Mme de Sévigné jouit auprès de ses contemporains ${ }^{34}$. Soulignons d'ailleurs que ces deux sources de légitimation des remontrances adressées à Mme de Grignan sont largement conditionnées par la condition de veuve de la marquise, qui lui octroie non seulement une totale autonomie en matière financière, mais encore une autorité exclusive sur ses enfants.

\section{Un type d'elocutio}

7 Lorsque le mode de relation des interlocuteurs le permet, « alors on peut proceder tout ouvertement ${ }^{35} »$, c'est-à-dire faire des remontrances de manière directe, sans avoir besoin de recourir aux précautions, largement topiques, dont se servent par exemple Mme de Sévigné et son fils Charles pour tempérer les critiques qu'ils adressent (l'un à la suite de l'autre, au sein d'une lettre à la comtesse) au Chevalier de Grignan, à qui ils reprochent les dépenses excessives engagées pour l'équipement de colonel du jeune 
marquis Louis-Provence. Dans cette lettre à deux énonciateurs, la marquise et son fils Charles, Mme de Sévigné pour sa part accumule les louanges, agrémente ses réprimandes d'une citation tirée d'une pièce de théâtre et pratique une autodérision propre à relativiser la virulence de son propos :

Je viens à vous, Monsieur, et je réponds à votre réponse, et je vais vous gronder, moi qui vous honore, moi qui vous estime, moi qui fais de vos jugements toutes mes décisions; je vous gronde pourtant. Eh ! d'où vient que vous laissez tailler en plein drap M. de Pracontal sur l'équipage de votre neveu ? Pourquoi, non M. de Grignan mais vous, comme sortant de cette place, ne décidez-vous pas sur ce qui est nécessaire? Ne voyez-vous pas bien qu'un homme qui est gâté par les vastes idées des grands Adhémar doit tout jeter par les fenêtres, et ne doit rien trouver de trop grand? Mais vous, la sagesse même, que n'avez-vous dit de ce petit colonel, comme Andromaque :

\section{Il est du sang d'Hector, mais il en est le reste?}

Et sur cela que n'avez-vous fait un équipage proportionné à celui des autres, à la misère du temps, au retranchement que l'on ordonne et dont le Roi donne l'exemple? Pourquoi n'avez-vous pas défendu le superflu, comme le Roi défend la vaisselle d'argent ? Pourquoi les quatre mille francs destinés à cette vaisselle ont-ils été engloutis encore dans cet équipage? Que n'ont-ils tenu lieu dans l'argent comptant qu'il faut qu'il emporte? Enfin pourquoi souffrez-vous que, quand cet équipage est déjà trop grand, cette pauvre Mme de Grignan donne encore ses deux mulets, et démonte sa litière, dont il me semble qu'on a toujours affaire et qui est si nécessaire en Provence? Enfin pourquoi songez-vous aux Adhémar quand vous savez le fond de leur sac? Mais je me laisse emporter au plaisir de grêler sur vous de deux cents lieues loin. C'est un plaisir qu'on ne prend guère en présence; j'ai profité de l'occasion une bonne fois [...].

Je vous souhaite une bonne santé. Hélas! quel plaisir de vous revoir comme autrefois! Je le souhaite passionnément, et vous demande, Monsieur, la continuation d'une amitié qui fait l'éloge de ceux à qui vous l'accordez. (19 avril 1690 : III, 866)

De son côté, et dans la même lettre, Charles, après une habile transition visant à amadouer son destinataire, veille non seulement à encadrer ses réprobations de vives protestations d'amitié et à les faire suivre de blandices concernant la carrière passée du Chevalier, mais encore à en atténuer le caractère péremptoire au moyen de modalisateurs et d'euphémismes :

J'ai bien peur, Monsieur, par cette raison, que vous ne fassiez ma satire et point du tout mon éloge. Cependant, comme on aime à se flatter, je veux croire que vous reconnaissez, par quelque sorte d'amitié, tous les sentiments que j'ai pour vous et, sur cela, je prends les douceurs que vous me dites pour beaucoup plus que vous ne les avez-vous-même données. Il est vrai que l'équipage du marquis de Grignan m'a un peu surpris. Je le trouve fort au-dessous des Adhémar, mais bien grand pour un jeune colonel ; je n'en ai point vu, ce me semble, de cette force, et je me souviens d'avoir vu jadis un Adhémar, très honorable, très habile, cadet à la vérité, mais aussi brigadier, dont cet aîné aurait pu suivre l'exemple, sans crainte qu'on lui eût disputé son nom, principalement depuis que M. d'Aiguebonne a perdu son procès.

Adieu, Monsieur. Personne ne vous honore assurément plus que moi. Madame ma femme en dit autant, et se plaint de votre retenue.

Enfin, comme si ces différentes parades ne suffisaient pas, Mme de Sévigné prend soin de «fini $[r]$ le ton des reproches » (id.) en faisant suivre ces deux séquences de passages empreints d'ingéniosité badine.

Dans les lettres à sa fille, au contraire, la marquise ne s'embarrasse guère d'assortir ses remontrances d'artifices oratoires qui sont alors monnaie courante, revendiquant 
même la franchise qui lui fait renoncer à sa "discrétion " pour ne "retenir ni [s]on affection, ni [s]a plume [sur] ces sortes de chapitres" (3 juillet 1675: I, 747) et " parle[r] sur ces chapitres à cœur ouvert » (24 mai 1690 : III, 883). De cette parrhésie assumée découle le choix de Mme de Sévigné non seulement, pour reprendre les termes de Puget de la Serre, de « decri[re] » la faute (certes de manière aussi rapide et allusive que possible), mais encore de l'« exagger[er] ${ }^{36} »-$ les termes rhétoriques d'exagération et d'amplification étant souvent employés, moyennant quelques flottements terminologiques, comme synonymes ${ }^{37}$. Destiné à faire prendre conscience à Mme de Grignan du caractère répréhensible de dépenses qui contribuent à " gâter encore [ses] affaires en Provence, au lieu de les raccommoder » (17 janvier $1680:$ III, 796), le recours systématique à l'amplification (comprise comme une forme d'insistance, de soulignement) révèle le caractère polémique des remontrances maternelles. Si Mme de Sévigné s'attache à «mettre devant les yeux » de la comtesse "sa faute, l'exaggerant vivement ${ }^{38}$ ", c'est parce que les correspondantes n'analysent pas la situation de la même manière: tandis que Mme de Grignan ne croit pas que renoncer aux frais de représentation suffira à rétablir un équilibre financier définitivement compromis par l'accumulation de dettes déjà anciennes, Mme de Sévigné se montre convaincue que sa fille pourrait être «la restauratrice de cette maison de Grignan» (21 juin 1671: I, 277), si seulement elle s'astreignait à réduire son train de vie (« Vous êtes maitresse de tout, pourvu que vous teniez un peu bride en main pour la dépense [...]», 8 juillet 1685 : III, 211). Ainsi s'explique le caractère franc et direct de remontrances qui visent à représenter à la comtesse l'énormitée ${ }^{39}$ de ses erreurs - c'est-à-dire à lui faire réévaluer la gravité de ses agissements.

\section{Une source de pathos}

Mme de Sévigné tâchant de persuader sa fille que la plupart de ses dépenses de prestige, aussi inévitables qu'elles puissent paraître au comte et à la comtesse, constituent de dangereux gaspillages, il n'est guère surprenant que ses remontrances fassent largement appel à l'amplification - envisagée, dans le sillage cicéronien, comme « une plus abondante preuve, \& un plus fort argument de ce qu'on a déjà dit, qui se donne créance dans l'esprit en émouvant la volontét0 ${ }^{2} »$. Rendre le propos plus fort, lui donner plus de poids afin d'accroître, grâce aux émotions sollicitées, son efficacité persuasive: tel est l'effet produit par le recours à l'amplification, qui constitue la principale source de pathos des remontrances à Mme de Grignan. On l'aura compris, loin d'être conçue selon la logique quantitative qui prévaut à l'heure actuelle, l'amplification est ici appréhendée à la lumière d'une tradition rhétorique attentive à ne pas la réduire à un dispositif visant à augmenter, à étendre et à développer la matière verbale ${ }^{41}$. Dans sa correspondance avec sa fille, Mme de Sévigné ne saurait en effet user des moyens volontiers artificiels que les ouvrages d'art oratoire préconisent afin d'assurer l'expansion du propos. Tournant le dos aux techniques d'amplification dont le caractère ostentatoire et véhément s'avère incompatible avec le genre de la lettre familière, Mme de Sévigné met en œuvre la forme d'amplification la plus concise qui soit, puisque circonscrite au niveau du $\operatorname{mot}^{42}$. Qu'un choix dénominatif consistant à employer un terme plus fort et plus insistant constitue un authentique procédé amplificatoire est d'ailleurs une idée que l'on retrouve dans plusieurs traités de 
rhétorique - qui incluent notamment parmi les supports de l'amplification la synonymie, l'hyperbole et la métaphore ${ }^{43}$. métaphorique constitue la solution à la difficulté de savoir comment « exagger[er] » la «faute» (selon la formule de Puget de la Serre) sans pour autant tomber dans une expansion verbale qui, en indisposant la destinataire, serait préjudiciable à l'efficacité des remontrances. En voici quatre exemples significatifs (nous soulignons chaque fois). Le jeu de hoca est présenté comme « un coupe-gorge »:

Mais à propos d'écus, quelle folie d'en perdre deux cents à ce chien de hoca! un coupe-gorge qu'on a banni de ce pays-ci, parce qu'on y fait de furieux voyages. Vous jouez d'un malheur insurmontable, vous perdez toujours. Voilà bien de l'argent qui vous épuise ; je ne puis croire que vous en ayez assez pour ne vous point sentir de ces pertes continuelles. ( 9 mars $1672: I, 455$.)

La capacité de $\mathrm{M}$. de Grignan à contracter de nouveaux emprunts pour acheter meubles et tableaux, en dépit de ses dettes criantes, est dite de la « magie noire ${ }^{44} »$ :

Vous me peignez Grignan d'une beauté surprenante ; eh bien ! ai-je tort quand je dis que M. de Grignan, avec sa douceur, fait toujours précisément tout ce qu'il veut? Nous avons eu beau crier misère; les meubles, les peintures, les cheminées de marbre n'ont-elles pas été leur train? Je ne doute point que tout cela ne soit parfaitement bien ; ce n'était pas là notre difficulté. Mais où a-t-il pris tant d'argent, ma fille? c'est la magie noire. (21 juin 1676 : II, 325.)

Le laxisme financier du comte et de la comtesse est comme «la bride abattue de l'équipage » :

Vous vous sacrifiez dans votre jeunesse à l'austérité de ces devoirs. Vous quittez le monde et la cour ; vous quittez même une place unique, qui contente l'orgueil, où vous êtes adorée - j'aime cette circonstance - et vous êtes à Grignan, occupée de ce qui peut être bon à votre maison et à votre fils. Je fais souvent de cet état le sujet de mon admiration, mais je blâme toujours un peu la bride abattue de l'équipage. Vous êtes dans votre château, où vous dites que vous ne dépensez pas trois sols; c'est ce que je ne crois pas. Vous y vivez trop honorablement. (21 mai 1690 : III, 880.)

Enfin, leurs déboires sont des « orages » et des « naufrages » :

Il me semble, ma bonne, que vous faites une jolie vie à Grignan. Malgré tant d'orages et tant de naufrages, je n'y vois que de l'abondance et de la magnificence; il est agréable de ne laisser pas de raccommoder ainsi ses affaires et de laisser couler le temps. Mais qui paie les cruels mécomptes de vos avances sur votre trésorerie ? (26 avril 1690 : III, 873.)

Dans ces quatre exemples, Mme de Sévigné trouve le moyen à la fois d'accroître la force argumentative de son propos (l'assimilation métaphorique du hoca à un "coupegorge ", des dépenses du comte alors qu'il n'a plus d'argent à de la " magie noire », du refus des Grignan de faire des économies à « la bride abattue de l'équipage » et de leurs revers à des « orages » et des "naufrages » forçant la destinataire à en percevoir le caractère dangereux) et de se prémunir contre la tentation d'un didactisme susceptible d'entraîner une rebuffade de la part de la comtesse (l'effet d'évidence propre à la fusion entre le thème et le phore ${ }^{45}$ dispensant l'épistolière de fastidieuses démonstrations sur la conduite inconsciente et irresponsable de sa fille).

11 Aussi courantes soient-elles (la plupart étant largement lexicalisées), les métaphores utilisées dans les séquences de remontrance, en déployant des connotations dysphoriques aussi violentes qu'indiscutables, fonctionnent comme des hyperboles dotées d'un puissant rendement pédagogique. De fait, si Mme de Sévigné éprouve le besoin de recourir à l'amplification métaphorique, c'est pour rectifier l'opinion que Mme de Grignan a de ses dépenses : plus la comtesse a tendance à en sous-estimer voire 
à en minimiser les dommages, plus sa mère se sent obligée de les exagérer au moyen de métaphores qui ont pour effet d'imposer une autre manière d'appréhender les choses. À y regarder de près, les métaphores nominales employées au sein des remontrances maternelles sont d'autant plus efficaces qu'elles apparaissent principalement au sein de deux configurations syntaxiques qui en accroissent la fonctionnalité pathétique - les métaphores visant à susciter en Mme de Grignan de la crainte (passion traditionnellement associée au genre délibératif) à l'égard des conséquences de ses erreurs de gestion, afin qu'elle en tire les conséquences pratiques.

La première tournure propre à renforcer la capacité qu'ont les métaphores nominales à imposer à la destinataire une autre façon de voir repose sur un «substantif relié à un autre par l'intermédiaire d'un verbe attributif ${ }^{46} \%$. Si la relation attributive est particulièrement appropriée pour inciter Mme de Grignan à changer de regard sur ses dépenses, c'est en vertu de la similitude formelle entre d'une part, les énoncés classificatoires, qui sont des phrases analytiques, et d'autre part, les énoncés métaphoriques, qui sont des phrases synthétiques ${ }^{47}$ - similitude qui a pour effet de présenter comme une évidence incontestable la relation d'identité instaurée par la relation attributive, comprise comme "un certain type d'identification définitoire qui consiste à "poser" un "objet" de discours et à dire ce qu'il est ${ }^{48}$ ". Dans les lettres à Mme de Grignan, le rendement cognitif des constructions métaphoriques attributives est encore accru par «la nature du prédéterminant de l'attribut substantival» - en l'occurrence, l'article indéfini, qui, parce qu'il est « le signe d'une coupure par rapport à la situation énonciative ${ }^{49}$ », fait que "l'attribut tend vers une valeur générique, classificatoire $^{50} »$.

Lorsqu'elle affirme que «la dépense d'Aix est une furie » et que la prise en charge de la nourriture des "bêtes et gens » lui "paraît [...] un fleuve qui entraîne tout », Mme de Sévigné exploite pleinement l'efficacité persuasive d'une telle configuration :

La dépense d'Aix est une furie; je me figure que vous êtes un peu revenue de l'économie de Grignan, où vous trouviez que vous pouviez vivre pour rien. Cela s'appelle rien, rien du tout: vos trois tables fort souvent dans la galerie, et toutes les visites et les trains; toujours nourrir bêtes et gens, chose qu'il n'y a plus que vous au monde qui fassiez. Toute cette fameuse auberge, tout ce concours de monde me paraît, quoi que vous disiez, un fleuve qui entraîne tout. (6 novembre 1680 : III, 53. Nous soulignons.)

L'épistolière se contente d'ailleurs rarement du « schéma syntaxique (Préd.1 + N1) + V. attr. + (Préd. 2 + N2) », optant souvent pour la mise en relief du terme métaphorique grâce au clivage ${ }^{51}$. La structure clivée c'est $N 1$ que N2 (obtenue par l'extraction d'un constituant placé en tête de phrase et encadré par la locution identifiante c'est et le pronom relatif que) présente l'avantage de sembler exprimer moins un jugement sèchement rationnel qu'une réaction émotionnelle - qu'il s'agisse d'identifier « la vie » que mènent à Aix le comte et la comtesse au « carnaval ",

Un hiver est impraticable à Grignan et très ruineux à Aix de la manière dont les jeux et les plaisirs sont à votre suite; c'est proprement le carnaval que la vie que vous faites. (21 février 1680 : II, 842. Nous soulignons.)

ou « la consommation de mille choses » à " un gouffre » :

Quand je considère votre château, ma bonne, rempli de toute votre grande famille, et des plaisirs qu'y attire M. de Grignan, je ne comprends pas que vous puissiez éviter d'y faire une fort grande dépense. Il n'y a point de provisions dont on ne trouve très promptement la fin avec tant de monde. C'est une affaire d'en racheter ; c'est un gouffre que la consommation de mille choses qu'il faut acheter. Cela n'était point ainsi du temps de feu monsieur votre beau-père, et je ne puis concevoir le château 
de Grignan comme un lieu de rafraîchissement pour vous. Ainsi l'intérêt continuel que je prends à vos affaires, ma bonne, ne me laisse point jouir du repos que je me suis imaginé dans ce lieu, où vous croyez toujours que vous vivez pour rien. C'est où il n'y a pas de remède. (3 juillet 1680 : II, 995 . Nous soulignons.)

Comme le souligne à la fin de ce passage l'épistolière elle-même (« vous croyez toujours que vous vivez pour rien »), son but est de rectifier la fausse perception que la comtesse a de ses dépenses - un but que sert précisément la "définition métamorphosante » qui résulte de «l'identification analogique ${ }^{52}$ » instaurée par la relation attributive, en dépit du caractère conventionnel des images utilisées.

La deuxième forme d'actualisation syntaxique des métaphores qui a pour effet d'en intensifier la valeur argumentative consiste en l'utilisation de l'anaphore démonstrative - qui, on le sait, "se prête [...] à "reclassifier" le référent, à le renommer. Elle attire l'attention sur un nouvel aspect de l'objet, dénommé autrement, ou présenté sous un nouveau point de vue ${ }^{53}$ ». Parce qu'elles ont la capacité d'imposer avec autant de discrétion que d'efficacité le jugement axiologique de l'épistolière, les groupes nominaux anaphoriques constituent l'un des moyens privilégiés des réprobations maternelles. En voici deux exemples. Mme de Sévigné présente les occupations de la comtesse comme un "tourbillon » aussi nuisible à sa santé qu'à ses affaires :

Mais ne pourriez-vous jamais faire quelque autre voyage où vous ne fussiez point dans cet horrible tourbillon, où vous puissiez jouir du repos qu'on trouve dans un si beau pays et de la société des personnes raisonnables que vous y avez? N'y pourriez-vous point un peu mieux dormir, c'est-à-dire simplement dormir ? car vous n'en avez pas le temps. Faut-il avoir toujours cette occupante et ruineuse et continuelle bassette? (26 juin 1689 : II, 626. Nous soulignons; la «bassette » est un jeu de cartes alors très en vogue, qui finira par être défendu tant il était ruineux.)

Dans une autre lettre, Mme de Sévigné a par deux fois recours au procédé. Les frais engagés pour les réceptions sont d' "effroyables débris et abîmes de toutes provisions » ou encore les dépenses au jeu sont des « petites pluies qui mouillent fort bien » :

Il y a du déchaînement au débordement des visites qu'on vous fait cette année; c'est comme par gageure: deux tables de douze couverts chacune dans cette galerie. C'est moi qui en suis cause en vous parlant de celles de M. de Chaulnes. Mais, ma bonne, il faut des lits dans la galerie; enfin cela me paraît dans un tel excès que je crois votre dépense très considérable, et quand vous me dites qu'on ne dépense rien à Grignan, ah! il est vrai, je ne manquerai pas de le croire. Nous savons bien ce que c'est ces effroyables débris et abîmes de toutes provisions. Et le jeu, pensez-vous que je croie que vous ne perdiez rien et M. de Grignan et vous? Je suis assurée que cela passe la dépense ordinaire. Nous connaissons ces petites pluies qui mouillent fort bien. Mais, ma bonne, il y des gens qui sont nés pour dépenser partout, comme il y en a qui se cassent la tête. Il n'y a aucun lieu de repos pour eux, ni qui puisse les ressuyer. Ils attirent le monde, la dépense, les plaisirs, comme l'ambre attire la paille. Il faut bien s'y résoudre, et monter dans le carrosse à quatre chevaux sans postillon. (11 septembre 1680 : III, 9. Nous soulignons.)

Rendues fortement pathétiques par leur nette connotation négative, les expressions démonstratives permettent ainsi à l'épistolière d'aiguiller sa destinataire sur la voie d'une reconfiguration cognitive tout en lui évitant de recourir aux algarades et aux semonces.

Indéniablement, l'option qui consiste à exagérer la faute au moyen de métaphores constitue, en raison de son caractère systématique, le principal marqueur stylistique des séquences de remontrance. Faire la plus forte impression possible dans l'esprit de Mme de Grignan en la forçant à entrevoir «l'abîme où conduit la grande dépense » 
(20 mai 1672 : I, 513), c'est-à-dire lui faire suffisamment peur pour qu'elle prenne enfin de bonnes résolutions: telle est la fonction de l'amplification métaphorique, qui constitue la principale source de pathos des réprimandes maternelles. Si ces métaphores servent aussi efficacement la stratégie de persuasion d'une mère qui n'hésite pas à accuser sa fille « de s'abîmer et de s'enfoncer à perte de vue » ( $1^{\text {er }}$ février 1690 : III, 824), c'est précisément parce qu'au lieu de s'adresser à la raison au moyen d'arguments toujours susceptibles d'être réfutés, elles visent à provoquer une réaction passionnelle propre à « émouvoir la volonté » - ce qui est le propre de l'amplification ${ }^{54}$.

Pour conclure, nous dirons qu'assurément, dans l'idéal, «c'est la marque d'un grand merite, que de souffrir modestement les remontrances", et que les personnes réprimandées « devraient savoir bon gré aux gens qui veulent les empêcher de faire des fautes, \& qui sont assez de leurs amis, pour leur ouvrir les yeux sur les irregularitez de leur conduite ${ }^{55} »$. Pourtant, au moment de formuler ses mises en garde, Mme de Sévigné est trop consciente du risque d'indisposer voire de heurter sa correspondante pour ne pas tenter de leur conférer une force persuasive qui soit néanmoins compatible avec la discrétion, la modestie et la délicatesse requises en une situation de discours aussi périlleuse. En choisissant comme technique d'exagération de la faute l'amplification métaphorique, conçue comme «augmentation [qui] se fait par un seul mot $^{56}$ ", Mme de Sévigné trouve le moyen à la fois d'ébranler l'opinion de sa destinataire au moyen de réprimandes dotées d'une forte densité pathétique sans tomber dans une grandiloquente abondance verbale ; de l'amener à changer sa façon de voir sans en passer par de pesants développements didactiques; de l'inciter à modifier sa conduite sans recourir à des admonestations frontalement prescriptives; de lui faire admettre la gravité de la situation sans employer un ton explicitement comminatoire ; de lui faire prendre conscience de ses erreurs d'appréciation sans faire preuve d'une véhémence ouvertement polémique. À cet égard, le choix de faire reposer l'efficacité des réprobations sur le recours systématique à la métaphore apparaît comme une authentique stratégie discursive - une stratégie qui confère aux séquences de remontrances des lettres à Mme de Grignan un fonctionnement discursif largement idiolectal.

\section{NOTES}

1. C. Lignereux, «La douceur comme stratégie de conciliation épistolaire ", Cahiers du Gadges, $\mathrm{n}^{\circ}$ 11, « L'art de la conciliation », S. Cornic et M. Servet dir., Genève, Droz, 2013, p. 173-188.

2. C. Lignereux, "Le conseil, un acte de langage contraire aux bienséances ? ", Cahiers du Gadges, $\mathrm{n}^{\circ} 10$, «Impertinence générique et genres de l'impertinence ( $\mathrm{XVI}^{\mathrm{e}}-\mathrm{XVIII}{ }^{\mathrm{e}}$ siècles) », I. Garnier et O. Leplatre dir., Genève, Droz, 2012, p. 451-460.

3. Les références données entre parenthèses mentionnent la date de la lettre et sa pagination (tome et page) dans l'édition de référence : Correspondance, éd. R. Duchêne, Paris, Gallimard, «Bibliothèque de la Pléiade ", 1972-1978, 3 tomes.

4. Sur l'exaltation du sens de l'économie de la marquise, présentée comme l'archétype de la bonne ménagère bourgeoise par les nombreuses éditions $d u X^{\mathrm{e}}$ siècle destinées à la jeunesse, 
voir Fr. Nies, Les Lettres de Mme de Sévigné. Conventions du genre et sociologie des publics [1972], Paris, Champion, coll. « Lumière classique », 2001, p. 238-243.

5. Condamner la lâcheté qui consiste à se taire plutôt que d'exprimer sa désapprobation : tel est l'objet de l'intervention de Clearque (dans M. de Scudéry, « De la complaisance », Conversations sur divers sujets, t. I, Paris, Cl. Barbin, 1680, p. 326) : «J'entens par une complaisance muette, reprit Clearque, ces Gens, qui de peur de dire quelque chose qui déplaise à leur Amis, leur laissent faire cent sotises, sans les en avertir : \& qui ne sçavent pas, que la derniere marque d'amitié, est de donner un avis fidelle. » Un peu plus loin dans la conversation (ibid., p. 330-331), Clearque insiste sur le fait que si « la complaisance est sans doute, le lien de la societé civile, [...] elle ne doit jamais, comme je l'ay déjà dit, ny trahir, ny flater. "

6. J.-B. Morvan de Bellegarde, Réflexions sur la politesse des mœurs, Amsterdam, H. Desbordes, 1699, p. 277 : «La politesse ne permet pas toûjours de reprendre ceux qui font des fautes; mais il y a de certaines conjonctures, où l'honneur, le devoir, l'amitié l'exigent absolument: c'est un rôle difficile à faire ; il faut avoir bien de la delicatesse pour se ménager avec ses amis qui ont fait quelque fausse démarche; mais ce seroit aussi une espece de cruauté de les abandonner à leur mauvaise conduite."

7. Telle est l'interprétation défendue par J. Duchêne, Françoise de Grignan ou le mal d'amour, Paris, Fayard, 1985.

8. Antoine Furetière, Dictionnaire universel, La Haye-Rotterdam, Arnout et Reiner Leers, 1690, s. v. advis (tout début de l'entrée).

9. Attentifs à définir un bon usage des conseils (le terme, d'emploi lâche, étant alors doté d'une valeur générique sensible), les auteurs de manuels épistolaires insistent sur la nécessité de n'en formuler que lorsque les « occasions » l'exigent. Tel est le cas de P. Richelet à propos des « Lettres d'Amitié » (dans Les plus belles lettres françoises sur toutes sortes de sujets, Tirées des meilleurs Auteurs, avec des Notes, Basle, J. Rodolf Tourneisen, 1752, t. I, « Observations sur l'art d'écrire des lettres », p. XVIII) : «Il ne faut être ni avare, ni prodigue de ses Conseils : mais il est bon de les donner avec quelque ménagement. Si l'amitié ne permet pas de les refuser à un ami qui en a besoin, elle veut qu'on juge de lui favorablement \& qu'on lui croye assez d'esprit pour se gouverner soi-même. Il y a des occasions où l'on peut passer sur ce scrupule, comme lors qu'il s'agit d'une chose trèsimportante \& dont notre ami n'est pas à portée de connoître aussi-bien que nous toutes les conséquences. » Tel est également le cas de J.-L. de Grimarest, qui précise dans l'article qu'il consacre aux "Lettres familières" (dans son Traité sur la manière d'écrire des lettres et sur le cérémonial, La Haye, A. Moetjens, 1709, p. 46) : « Nous ne devons lâcher nos conseils que quand on nous les demande; ou dans les occasions essentielles, si l'on prévoit que la personne à qui l'on écrit en peut absolument avoir besoin. "

10. J. Puget de la Serre, Le Secrétaire à la mode, Amsterdam, L. Elzevier, 1646, p. 6 et p. 24.

11. Ibid., p. 11-12. Les définitions de Puget de la Serre sont encore reproduites telles quelles dans les manuels du début du siècle suivant, notamment ceux de R. Milleran (dans Le Nouveau Secrétaire de la Cour, ou Lettres familières sur toutes sortes de sujets, Paris, N. Le Gras, 1714, p. V-VI, et dans Le Nouveau Secrétaire du Cabinet, contenant des lettres sur différents sujets, Paris, T. Legras, 1719, p. 5-6).

12. Ibid., p.10-11. Alors que les traités épistolographiques en latin du siècle précédent (notamment celui d'Érasme en 1522, de J. Voellus en 1578 ou de M. Junius en 1608, cités plus loin) cultivent, à l'intérieur du genre judiciaire, des raffinements taxinomiques qui visent autant à l'exhaustivité qu'à la systématicité, les secrétaires du XVII siècle en langue vernaculaire non seulement renoncent à des distinctions rhétoriques inadaptées au public mondain, mais encore font le plus souvent disparaître ce que dans son article « Manuels épistolaires et identité sociale (XVI ${ }^{\mathrm{e}}$-XVIII ${ }^{\mathrm{e}}$ siècles)»( R.H.M.C., tome 40-4, octobre-décembre 1993, p. 545), M. Daumas désigne comme des « lettres agonistiques » - ce qui n'est guère surprenant dans la mesure où « de façon 
générale, l'image de l'identité sociale que donnent les secrétaires ne repose pas sur l'exclusion, le blâme, la critique. »

13. J.-B. Morvan de Bellegarde, op. cit., p. 120-121.

14. J. Puget de la Serre, op. cit., p. 10.

15. Voir les lettres du 9 mars 1672 (I, 455) ; du 7 juin 1675 (I, 727) ; du 9 octobre 1675 (II, 123).

16. Voir les lettres du 2 août 1671 (I, 310-311) ; du 6 novembre 1680 (III, 53) ; du 20 novembre 1689 (III, 758).

17. Voir les lettres du 24 juillet 1675 (II, 20); du 13 mai 1680 (II, 929); du 24 mai 1690 (III, 883).

18. Voir les lettres du 14 février 1689 (III, 503); du 2 mai 1689 (III, 590); du 14 septembre 1689 (III, 692).

19. Voir les lettres du 3 juillet 1675 (I, 747) et du 5 juin 1680 (II, 962).

20. Le terme de répréhension est défini par le dictionnaire de Furetière comme "Correction, reprimende d'un superieur» et par celui de l'Académie comme "Réprimande, blâme, correction ». Les traités de rhétorique contemporains classent les répréhensions dans le genre judiciaire. Citons R. Bary (La Rhétorique Françoise, où pour principale augmentation l'on trouve les secrets de nostre langue, Paris, Pierre Le Petit, 1665, p. 227, nous soulignons) : " « On range sous le genre judiciaire, les reproches, les excuses, les reprehensions, les libelles diffamatoires, \& les apologies ». Citons également J. du Roure (La Rhétorique françoise, Paris, Chez l'auteur, 1662, p. 80, nous soulignons) : « «Les Invectives, les Menaces, les Reprehensions, les Reproches et les Excuses sont des accusations ou des deffenses, contenuës consequemment sous le Genre Judiciaire, dont elles suivent les préceptes. »

21. J. Puget de la Serre, op. cit., p. 10.

22. Furetière, s. v. remonstrance. Cet objectif d'amendement constitue l'un des critères distinctifs de ce que les traités de rhétorique nomment l'objurgatio ou objurgation. C'est ainsi que pour N. Caussin, «Est enim ferme objurgatio superioris ad inferiorem, ob delicta quaedam reprehensio, spectans emendationem ejus, qui arguitur» (Eloquentiae sacrae et humanae parallela. Libri XVI, Paris, S. Chappelet, 1619, p. 543, nous soulignons: «L'objurgation se fait presque du supérieur à l'inférieur, elle est une sorte de répréhension d'une faute, avec pour but l'amendement de celui qu'elle attaque »); que pour G.-J. Vossius, "Objurgatio est reprehensio ob delictum, qua emendationem alterius sibi proponat» (Rhetorices Contractae, sive Partitionum oratoriarum. Libri V, Brunswick, C.-F. Zilliger, 1647, p. 212, nous soulignons : « L'objurgation est la répréhension d'une faute, qui se propose pour but l'amendement d'autrui »); que pour G. Pelletier, non seulement «Et est reprehensio, quae sibi proponit emendationem ejus, qui contra officium et honestos mores peccaverit [...] », mais encore "fructus Increpationis, ac finis est Emendatio» (Reginae Palatium Eloquentiae, Lyon, J.-A. Candy, 1653, p. 866 et 869, nous soulignons : "C'est une répréhension, qui se propose pour but l'amendement de celui qui aura péché contre le devoir et l'honnêteté », et « le résultat et la finalité des Reproches cinglants, c'est l'Amendement»). Sauf mention contraire, les traductions sont nôtres.

23. P. Jacob, Le Parfait Secrétaire, ou la manière d'escrire et de respondre à toute sorte de Lettres, par Préceptes \& par Exemples, Paris, A. de Sommaville, 1646, p. 407. De manière révélatrice, dans le modèle de lettre d'avertissement que donne Jacob, le locuteur, au moment où il accumule les arguments destinés à justifier le « langage qu'[il] tien[t] », utilise les termes de "remonstrances », de « reprimendes » et de « correction» (ibid., p. 412-413).

24. S'inspirant largement de la typologie d'Érasme, pour qui la monitoria epistola («lettre d'avertissement », « monitoire ») relève du genre délibératif (De Conscribendis Epistolis [1522], éd. J.-C. Margolin, dans Opera Omnia Desiderii Erasmi Roterodami, Ordinis Primis, Tomus Secundus, Amsterdam, North-Holland Publishing Company, 1971, p. 488 sq.), J. Voellus distingue plusieurs espèces de monitio (ou avertissement), ainsi que le résume la manchette «Praeceptiones, admonitiones, \& objurgationes ad deliberationem referuntur, \& unius monitionis nomine comprehenduntur ", « Enseignements, admonestations et objurgations relèvent du délibératif, et sont tous compris 
sous un seul terme, celui d'admonition [d'avertissement]». Il insiste sur le fait qu'il est difficile de distinguer l'objurgatio (la remontrance) de l'admonitio (l'avertissement), De Ratione conscribendi epistolas utilissimae praeceptiones, Lyon, J. Pillehotte, 1578, p. 31.

25. Bienveillance et amitié apparaissent comme les deux éléments constitutifs de l'ethos propre à l'objurgatio, dont le but est d'être utile au destinataire - ce qui la distingue de l'invective. Pour N. Caussin dans son Eloquentiae sacrae et humanae parallela (op. cit., p. 543), "Objurgatio differt ab invectiva, quod haec fit adversus inimicos, illa dilectos etiam attingit, Haec flagrans fit, \& tonans, illa acris quidem saepe, sed longe moderatior: Ad summum haec nocere ut plurimum cupiat, illa prodesse. » "L'objurgation diffère de l'invective, en ce que celle-ci se fait contre des ennemis, et celle-là attaque même des amis. Celle-ci se fait par l'incendie et le tonnerre, celle-là quoique souvent âpre est de loin plus modérée. En un mot, celle-ci en général désire nuire, et celle-là être utile. » Pour G.-J. Vossius (op. cit., p. 212), «Differt ab invectiva, quod haec fere circa inimicos versetur, \& id spectet, ut noceat : illa instituatur erga amicos, \& hoc studeat, ut prosit. " "L'objurgation diffère de l'invective, en ce que celle-ci s'adresse à des ennemis, dans le but de leur nuire : et celle-là, à des amis, et pour leur être utile. »

26. Les préceptes que donne P. Jacob pour la lettre d'avertissement dans Le Parfait Secretaire (op. cit., p. 410) montrent que l'efficacité de celle-ci est subordonnée au même éthos: « Nous dirons que nostre âge \& notre autorité nous ont acquis des experiences, dont il n'est pas encore capable ; que nous sommes tout prests à l'aider de nos fideles conseils. »

27. J. Puget de la Serre, op. cit., p. 10.

28. R. Amossy, article "Ethos », dans P. Charaudeau et D. Mainguenau dir., Dictionnaire d'analyse du discours, Paris, Seuil, 2002, p. 239.

29. Voir La Barre Matéï, L'Art d'écrire en François, ou la manière de faire des lettres, dans L'Art d'écrire en François, ou la manière de faire des Complimens, des Lettres, des discours en Dialogues, des Traductions, des Harangues, l'Histoire, des Romans, Paris, N. Jolybois, 1662. Si l'on se réfère aux préceptes de La Barre Matéi dans la rubrique intitulée " De la forme de commencer une lettre » (ibid., p. 11), la répréhension, comprise comme espèce d'avis, constitue non seulement un sujet courant des lettres destinées "à des personnes inferieures ", mais encore l'une des entrées en matière requises: "Quand on écrit à des personnes inferieures, il faut que le commencement leur donne quelque avis: quelque ordre que l'on recommande de suivre; ou leur fasse quelque reprehension agreable, ou les somme de faire quelque chose à quoy vôtre Lettre semble les engager. »

30. Ce critère définitoire constitue un autre point commun entre la remontrance et l'objurgatio. Pour G.-J. Vossius (op. cit., p. 212), « objurgatio plurimum est superioris : ut Imperatoris erga militem, patris adversus filium» ("l'objurgation en général est le fait d'un supérieur: par exemple, le Général envers le soldat, le père contre son fils»). La définition que donne G. Pelletier de l' objurgatio commence ainsi (op.cit., p. 866) : "Quoniam objurgatio fere semper est superioris ad inferiorem: ut Imperatoris ad milites: Principis ad subditos: Pastoris ad gregem: Parentis ad filios. » «Puisque l'objurgation est presque toujours le fait d'un supérieur s'adressant à un inférieur : par exemple, le Général à un soldat; le Prince à ses sujets; le Pasteur à son troupeau; le Père ou la Mère à ses fils. » De manière significative, G. Pelletier termine le développement consacré à l' objurgatio en donnant pour exemple le discours d'un père «à son fils qui dilapide son patrimoine » (« Objurgat pater filium patrimonium effundentem », ibid., p. 870).

31. Dictionnaire de l'Académie françoise, Paris, Veuve Jean-Baptiste Coignard, 1694.

32. R. Duchêne, «Une reconnaissance excessive ? Mme de Sévigné et son Bien Bon ", XVII siècle, $n^{\circ}$ 74, 1967, p. 27-53.

33. Nous renvoyons aux analyses biographiques de R. Duchêne, Mme de Sévigné ou la chance d'être femme, Paris, Fayard, 2002.

34. Dans le portrait qu'elle dresse de celle qu'elle nomme la princesse Clarinte (Clélie, éd. Ch. Morlet-Chantalat, Paris, Champion, 2003, p. 460), Mlle de Scudéry souligne que «Ce qu'il y a de merveilleux en cette personne, c'est qu'en l'âge où elle est, elle songe aux affaires de sa 
maison aussi prudemment que si elle avait toute l'expérience que le temps peut donner à un esprit fort éclairé ». De même, dans son Histoire généalogique de la maison de Rabutin (éd. P. Beaune, Dijon, E. Rabutot, 1866, p. 59-60), Bussy-Rabutin insiste sur le sens des responsabilités qui anime Mme de Sévigné, attentive aussi bien à surveiller l'équilibre de ses finances qu'à établir durablement ses enfants : «Par sa bonne conduite (je n'entends point icy parler de ses mœurs), je veux dire par sa bonne administration, elle augmenta son bien, ne laissant pas de faire la despense d'une personne de sa qualité, de sorte qu'elle donna un grand mariage à sa fille, et luy fit épouser François Adémar de Monteil, comte de Grignan, lieutenant pour le Roy en Languedoc et puis après en Provence. Les soins que Marie de Rabutin avoit pris de sa maison, n'y avoient pas seuls mis tout le bon ordre qui y étoit. L'abbé de Coulanges, son oncle, homme d'esprit et de mérite, l'avoit fort aidée à cela. "

35. J. Puget de la Serre, op. cit., p. 10.

36. On aura reconnu le double mouvement de la narration et de la confirmation (ou argumentation), tel qu'il est présenté par certains traités de rhétorique comme constitutif de l' objurgatio. Voir par exemple C. Pajot, Tyrocinium eloquentiae sive Rhetorica nova, et facilior [1647], Venise-Bassano, A. Remondini, 1708, p. 276 : «Narratio rem subjicit oculis per hypotyposim [...]. Confirmatio crimen exaggerat ab aliis convenientibus locis Rhetoricis. » « La narration met l'affaire sous les yeux en recourant à l'hypotypose [...]. La confirmation exagère le crime en tirant argument des autres lieux Rhétoriques appropriés à la situation. »

37. Pour Furetière, l'exaggeration désigne la «Figure de Rhetorique par laquelle on augmente ou on diminuë les choses, on dit plus ou moins qu'il n'y en a ", et exaggerer signifie "User d'hyperbole, parler des choses autrement qu'elles ne sont, soit pour les augmenter, soit pour les diminuer ou en bien, ou en mal». Le parallèle avec la définition que donne le dictionnaire de Richelet de l'amplification s'impose : «Terme de Rétorique. Adresse d'agrandir une chose, ou de la faire plus petite qu'elle n'est ".

38. Pour P. Jacob, c'est la première étape du protocole argumentatif propre à la lettre d'avertissement (op. cit., p. 407).

39. Le terme est notamment utilisé par R. Milleran à propos de la lettre «Pour avertir d'une faute " (conçue comme l'une des cinq «especes particulieres» des lettres familières), dans ses Lettres familières, galantes, Et autres sur toutes sortes de sujets, avec leurs Réponses (seconde édition, Bruxelles, J. Leonard, 1692, p. 411) : «Si vous avez de l'autorité sur celui que vous avertissez, ces sortes de lettres ne sont plus de civilité, car vous lui faites voir par des termes absolus qu'il ternit sa reputation; ensuite vous l'exhortez de quitter le vice, en lui representant son enormité, \& de le reparer par de belles \& de genereuses actions. » Les préceptes que donne La Barre Matéi à propos de la lettre de blasme, y recourent également (op. cit., p. 18-19) : "Pour blasmer quelqu'un, il faut exprimer nôtre deplaisir \& celuy des autres, touchant la faute qu'il a commise, luy en faire reprimande, par raisons \& avis legitimes faire des prieres à Dieu pour luy; \& luy faire voir l'enormité de la chose; le rappeller au droit chemin, s'il est possible. » Il en va de même pour le modèle proposé (ibid., p. 18-19) : «Souffrez que je vous âvertisse de vôtre devoir, \& que je vous remontre l'énormité de vôtre faute ». Nous soulignons.

40. Telle est la traduction que donne J. Leven de Templery de la définition cicéronienne de l'amplification (L'Eloquence du temps, enseignée à une dame de qualité, Paris et Bruxelles, Jean Leonard, 1707, p. 239-240). Rappelons la formule originale des Partitiones oratoriae (Divisions de l'art oratoire, éd. H. Bornecque, Paris, Les Belles Lettres, [1924], 2002, § 53, p. 21) : « Est igitur amplificatio gravior quaedam affirmatio, quae motu animorum conciliet in dicendo fidem. " "L'amplification est donc une sorte d'affirmation plus forte, qui, en touchant les âmes, doit rendre le discours convaincant. »

41. Sur la divergence entre la perception quantitative de l'amplification qui domine à l'heure actuelle et la tradition rhétorique sous-jacente aux théories classiques, nous renvoyons aux 
analyses de S. Macé, L'Amplification : entre rhétorique et stylistique, Mémoire d'Habilitation à Diriger des Recherches, 2008, inédit, p. 3-8.

42. Sur la distinction fondatrice que fait Quintilien, à la suite d'une allusion de Cicéron, entre amplificatio rerum et amplification verborum, telle qu'elle a souvent été reprise et retravaillée, certes de manière inégalement théorisée, par les traités de rhétorique de l'âge classique, voir S. Macé, ibid., p. 48-53.

43. L'idée que l'amplification puisse se localiser au niveau des mots provient de Cicéron (Divisions de l'art oratoire, op. cit., § 53, p. 21) et surtout de Quintilien (Institution oratoire, éd. J. Cousin, Paris, Les Belles Lettres, 1978, t. V, livre VIII, 4, p. 86 sq.). Parmi les traités de rhétorique qui mentionnent la synonymie, l'hyperbole et la métaphore, conçues comme des figures fondées sur le choix de mots plus forts, parmi les supports d'amplificatio verborum, S. Macé signale: L. Schenkel, Rhetorica Cornelii Valerii Ultrajectini, Anvers, ex officinam Plantinianam, 1593, p. 85 ; L. de Grenade, La Rhétorique de l'Eglise, ou l'Eloquence des Prédicateurs, Paris, J. Villette, 1698, p. 242-243 (ce texte est édité dans ce numéro, section «Atelier») ; Fr. Pomey (J. Jouvancy), Candidatus Rhetoricae, Paris, J. Barbou, 1712, p. 155 ; D. Colonia, De Arte Rhetorica Libri Quinque, Parme, Borsiana, 1762, p. 47-50.

44. Il s'agit là d'une expression courante, comme l'attestent les dictionnaires de l'époque. Pour l'Académie, « On dit proverbial. d'Une chose qu'il est malaisé de pénétrer, \& où l'on ne comprend rien, que C'est la magie noire». Quant à Furetière, il précise que le terme de magie "se dit figurément en Morale, des choses dont on ne peut avoir pleine connaissance, tant elles sont cachées. Les affaires de cet homme sont si brouillées, que c'est la Magie noire. La depence de la marine, de l'artillerie est une Magie noire, on ny voit goutte. »

45. Pour Ch. Perelman et L. Olbrechts-Tyteca (Traité de l'Argumentation. La nouvelle rhétorique [1958], Bruxelles, Éditions de l'Institut de Sociologie-Université Libre de Bruxelles, 1970, p. 535-536), l'efficacité argumentative de la métaphore est liée au fait qu'elle fonctionne comme « une analogie condensée, résultant de la fusion d'un élément du phore avec un élément du thème »- «l'analogie, grâce à cette fusion, [étant] présentée non comme une suggestion, mais comme une donnée. ».

46. I. Tamba, Le Sens figuré. Vers une théorie de l'énonciation figurative, Paris, PUF, 1981, p. 84.

47. Constatant que "le schéma syntaxique le N1 est un N2 [est] considéré à la fois comme la structure canonique des phrases classificatoires et des métaphores nominales », I. Tamba montre la nécessité de dépasser "leur identité structurelle apparente» en comparant leur fonctionnement sémantico-syntaxique ( La femme est-elle une fleur comme le bleuet est une fleur? Métaphore et classification: les structures en "Le N1 est un N2" », dans N. Charbonnel et G. Kleiber dir., La Métaphore entre philosophie et rhétorique, Paris, PUF, 1999, p. 206-209). Tandis que les phrases analytiques "expriment une vérité dépendant de leur sens et non des faits empiriques ", les phrases synthétiques requièrent une "validation [...] d'ordre empirique et donc variable suivant les contextes de référence » (ibid., p. 212).

48. I. Tamba, op. cit., p. 169.

49. Ibid., p. 84.

50. Ibid., p. 85. Voir également ibid.p. 169 : «Quand la valeur être-tel est affectée à un nom précédé d'un article indéfini, on a affaire à une occurrence spécifique d'une classe notionnelle qui va servir de définissant à l'occurrence d'une autre classe notionnelle à laquelle elle est identifiée référentiellement. On aura ainsi une définition, par identification analogique, de deux occurrences définies appartenant à des classes notionnelles différentes. »

51. Ibid., p. 84.

52. Ibid., p. 170.

53. A. Herschberg-Pierrot, Stylistique de la prose, Paris, Belin, 1993, p. 241.

54. Que le but de l'amplification soit moins de convaincre l'intelligence que d'émouvoir la volonté est une idée sur laquelle insiste particulièrement M. du Cygne dans le chapitre qu'il consacre à 
l'amplification - un chapitre largement structuré par l'opposition entre l'amplificatio verborum et les différentes sortes d'amplificatio rerum (Ars rhetorica, Cologne, W. Metternich, 1706, p. 72-84). Dès l'introduction de ce chapitre, l'auteur, après avoir cité la définition cicéronienne, précise (ibid., p. 72) : «Ex qua definitione adverte finem proprium ac proximum Amplificationis esse, magis voluntatem movere, quam intelligentiam convincere ", "À partir de cette définition, remarquez que la finalité propre et immédiate de l'Amplification est plus d'émouvoir la volonté que de convaincre l'intelligence." Dans l'article VI, intitulé «Usus Amplificationis, \& quomodo differat ab Argumentatione » (ibid., p. 83 : «De l'usage de l'Amplification, et de quelle manière elle diffère de l'Argumentation »), il souligne que l'argumentation et l'amplification se distinguent notamment par leur but: «Nam finis proprius \& proximus argumentationis est facere fidem, seu intelligentiam convincere: amplificationis vero movere voluntatem." «Car la finalité propre et immédiate de l'argumentation, c'est de faire ajouter foi à ce que l'on dit [fidem facere est une formule cicéronienne], ou si l'on préfère de convaincre l'intelligence : quant à l'amplification, son but est d'émouvoir la volonté. »

55. J.-B. Morvan de Bellegarde, op. cit., p. 120.

56. La formule est de Le Gras dans La Réthorique [sic] Françoise, ou les preceptes de l'ancienne et vraye eloquence: Accomodez à l'usage des conversations \& de la Société civile: Du Barreau: Et de la Chaire (Paris, chez l'auteur, 1671, p. 219-221 - sur Gallica). Le Gras commence ainsi l'énumération des six moyens (qu'il classe du plus concis au plus copieux) permettant de réaliser une amplification (ibid.) : "Premierement, l'augmentation se fait par un seul mot, comme d'appeler voleur un homme qui nous a fait quelque tort mediocre.»

\section{AUTEUR}

\section{CÉCILE LIGNEREUX}

Université Grenoble Alpes - RARE Rhétorique de l'Antiquité à la Révolution 